\title{
Introduction to tropical algebraic geometry
}

\author{
Diane Maclagan
}

\begin{abstract}
This is an expository introduction to tropical algebraic geometry based on my lectures at the Workshop on Tropical Geometry and Integrable Systems in Glasgow, July 4-8, 2011, and at the ELGA 2011 school on Algebraic Geometry and Applications in Buenos Aires, August 1-5, 2011.
\end{abstract}

\section{Introduction}

Tropical algebraic geometry is algebraic geometry over the tropical semiring (Definition 1.1). This replaces an algebraic variety by a piecewise linear object which can be studied using polyhedral combinatorics.

Tropical geometry has exploded as an area of research in the last decade, with many new connections and applications appearing each year. These include enumerative geometry, mirror symmetry, arithmetic geometry, and integrable systems. It builds on the older area of tropical mathematics, more commonly known as maxplus algebra, which arises in semigroup theory, computer science, and optimization. The name "tropical" was coined by some French mathematicians in honor of the Brazilian computer scientist Imre Simon. See 6] or [19 for an introduction to this older area.

The goal of this expository and elementary article is to introduce this exciting new area. We develop the theory of tropical varieties and outline their structure and connection with "classical" varieties.

There are several approaches to tropical geometry. We follow the "embedded" approach, which focuses on tropicalizing classical varieties. Another important branch of the subject focuses on developing an abstract theory of tropical varieties in their own right. See work of Mikhalkin and collaborators [29, [30] for details on this. This direction is most developed for curves. One of the attractions of tropical geometry is that it has so many disparate, but connected, facets, so any survey is necessarily incomplete.

We begin by introducing tropical mathematics, focusing on tropical polynomials and their solutions. In this paper we will follow the minimum convention for the tropical semiring:

2010 Mathematics Subject Classification. Primary 14T05; Secondary 14M25, 52B20, 12J25.

Partially supported by EPSRC grant EP/I008071/1. 


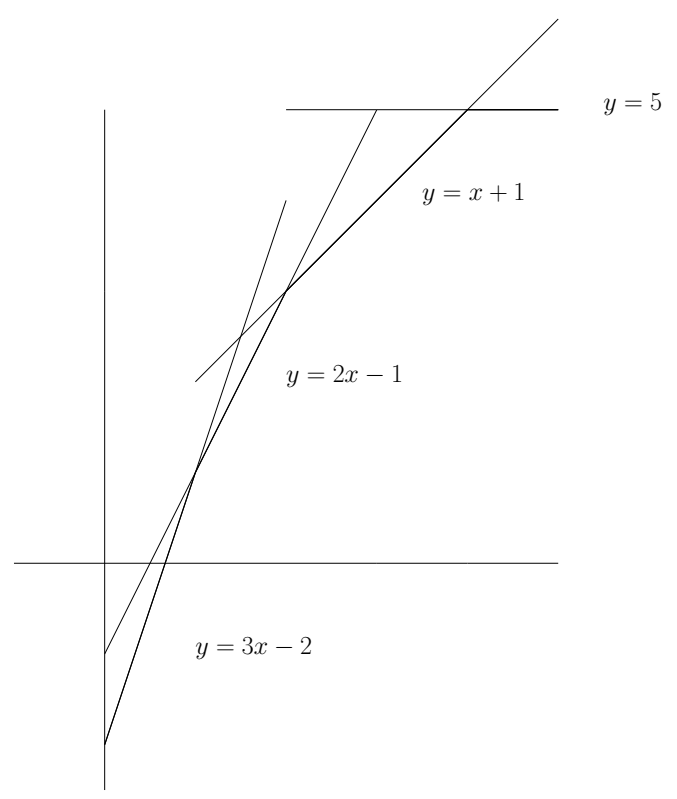

Figure 1. A tropical polynomial

Definition 1.1. The tropical semiring is $\mathbb{R} \cup\{\infty\}$, with operation $\oplus$ and $\otimes$ given by $a \oplus b=\min (a, b)$ and $a \otimes b=a+b$.

The tropical semiring is associative and distributive, with additive identity $\infty$ and multiplicative identity 0 . This satisfies every axiom of a ring except additive inverses, so is a semiring.

Tropical operations are often simpler than regular operations. For example we have the "Freshman's dream": $(x \oplus y)^{n}=x^{n} \oplus y^{n}$. The following examples illustrate that tropical polynomials are piecewise linear functions.

EXAMPLE 1.2. (1) The tropical polynomial $F(x)=-2 \otimes x^{3} \oplus-1 \otimes x^{2} \oplus$ $1 \otimes x \oplus 5$ is $\min (3 x-2,2 x-1, x+1,5)$ in regular arithmetic. This is the piecewise linear function whose graph is shown in Figure 1 .

(2) The tropical multivariate polynomial $x \oplus y \oplus 0$ is the piecewise linear function $\min (x, y, 0)$ in regular arithmetic. Note that the zero cannot be removed here, as zero is not the additive identity. This is a function from $\mathbb{R}^{2}$ to $\mathbb{R}$ whose domain is shown in Figure 2

With no subtraction, it is not obvious how to solve polynomial equations. For example, the equation $x \oplus 2=5$ has no solution. This problem has the following resolution.

Definition 1.3. The tropical hypersurface $V(F)$ defined by the tropical polynomial $F$ in $n$ variables is the nondifferentiable locus of $F$ in $\mathbb{R}^{n}$. This is the set of $x \in \mathbb{R}^{n}$ for which the minimum is achieved at least twice in $F(x)$.

Example 1.4. For the first polynomial of Example 1.2 $V(F)=\{1,2,4\}$. For the second, $V(F)$ is the union of the three rays shown on the right in Figure 2 


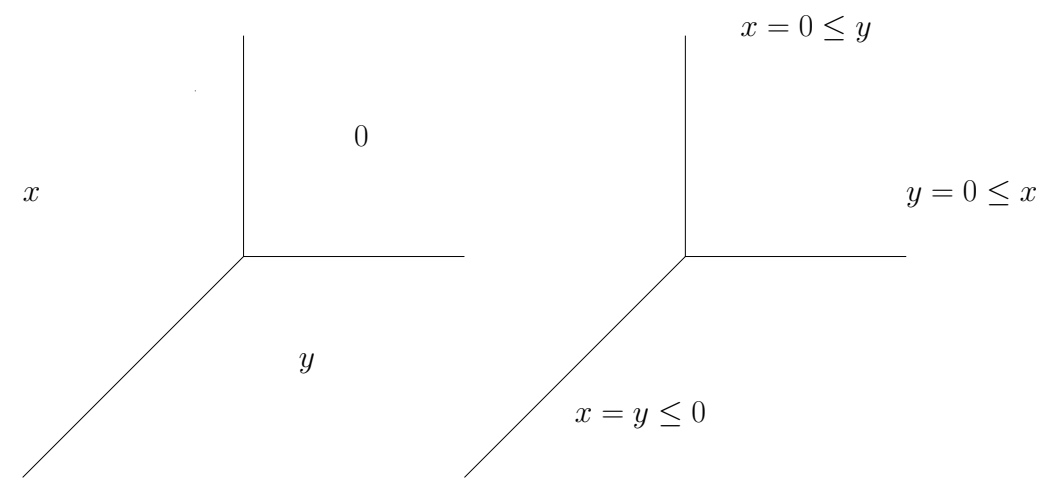

Figure 2. A tropical line

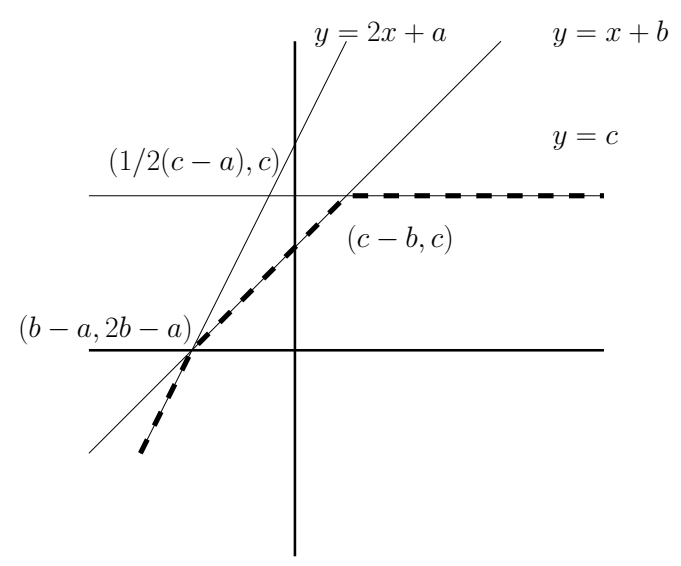

FiguRE 3. Tropical quadratic polynomials

EXAMPLE 1.5. The tropical quadratic formula is particularly simple. If $F(x)=$ $a \otimes x^{2} \oplus b \otimes x \oplus c$, then the graph of $F$ is shown in Figure 3. Note that there are two cases, depending on the sign of the tropical discriminant $a+c-2 b$. If $2 b \leq a+c$ then $V(F)=\{c-b, b-a\}$. If $2 b \geq a+c$ then $V(F)=\{(c-a) / 2\}$. Compare this with the usual quadratic formula, and the usual discriminant. Higher degree polynomials have similarly easy solutions.

\section{Tropical varieties}

The impact of tropical techniques comes when they are applied to "classical" objects, such as algebraic varieties. We now explain how to tropicalize certain algebraic varieties.

For a field $K$ we set $K^{*}=K \backslash\{0\}$. Fix a valuation val : $K^{*} \rightarrow \mathbb{R}$. This is a function satisfying:

(1) $\operatorname{val}(a b)=\operatorname{val}(a)+\operatorname{val}(b)$

(2) $\operatorname{val}(a+b) \geq \min (\operatorname{val}(a), \operatorname{val}(b))$.

Example 2.1. (1) $K=\mathbb{C}$ with the trivial valuation $\operatorname{val}(a)=0$ for all $a \in \mathbb{C}^{*}$. 


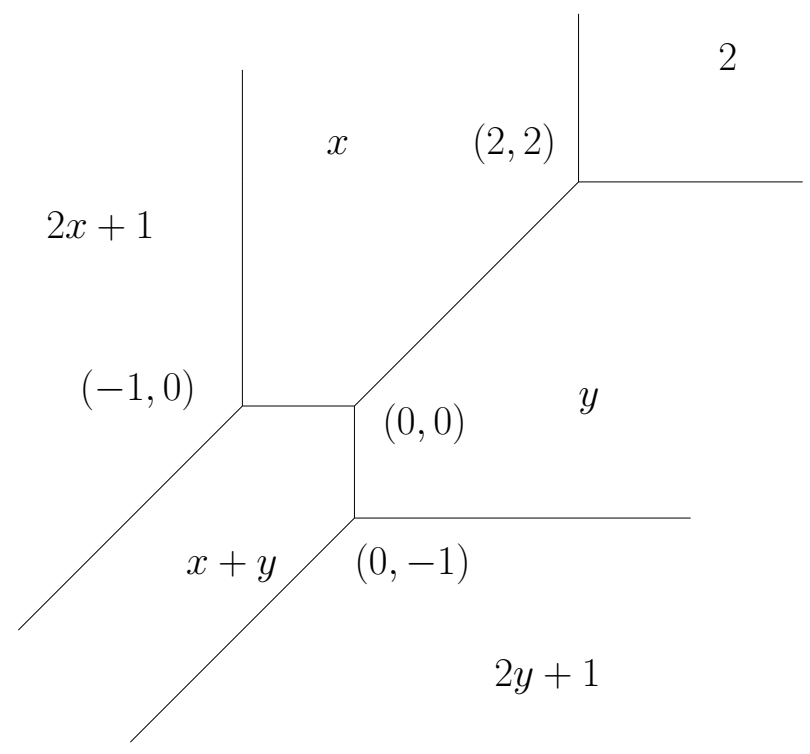

Figure 4. A tropical quadric

(2) $K=\mathbb{C}\{\{t\}\}$, the field of Puiseux series. This is the union $\bigcup_{n>1} \mathbb{C}\left(\left(t^{1 / n}\right)\right)$, and is the algebraic closure of the field of Laurent series. Elements are Laurent series with rational exponents where in any given series the exponents all have a common denominator. The valuation of $a \in \mathbb{C}\{\{t\}\}$ is the lowest exponent appearing. For example, $\operatorname{val}\left(3 t^{-1 / 2}+8 t^{2}+7 t^{13 / 3}+\ldots\right)=-1 / 2$.

(3) $K=\mathbb{Q}$ or $\mathbb{Q}_{p}$ with the $p$-adic valuation. If $q=p^{n} a / b \in \mathbb{Q}$ with $p$ not dividing $a$ or $b$, then $\operatorname{val}_{p}(q)=n$. For example, $\operatorname{val}_{2}(8)=3$, and $\operatorname{val}_{3}(5 / 6)=-1$.

Definition 2.2. The tropicalization of a Laurent polynomial $f=\sum c_{u} x^{u} \in$ $K\left[x_{1}^{ \pm 1}, \ldots, x_{n}^{ \pm 1}\right]$ is $\operatorname{trop}(f): \mathbb{R}^{n} \rightarrow \mathbb{R}$ given by

$$
\operatorname{trop}(f)(w)=\min \left(\operatorname{val}\left(c_{u}\right)+w \cdot u\right) .
$$

This is obtained by regarding the addition and multiplication as tropical addition and multiplication, and changing the coefficients to their valuations.

EXAMPLE 2.3. Let $K=\mathbb{Q}$ with the 2 -adic valuation, and let $f=6 x^{2}+5 x y+$ $10 y^{2}+3 x-y+4 \in \mathbb{Q}\left[x^{ \pm 1}, y^{ \pm 1}\right]$. Then $\operatorname{trop}(f)=\min (2 x+1, x+y, 2 y+1, x, y, 2)$. This is illustrated in Figure 4.

For $f \in K\left[x_{1}^{ \pm 1}, \ldots, x_{n}^{ \pm 1}\right]$ the (classical) hypersurface $V(f)$ equals $\left\{x \in\left(K^{*}\right)^{n}\right.$ : $f(x)=0\}$. The tropicalization $\operatorname{trop}(V(f))$ of $V(f)$ is the tropical hypersurface of $\operatorname{trop}(f)$. This is the nondifferentiable locus of $\operatorname{trop}(f)$, or equivalently:

$\operatorname{trop}(V(f))=\left\{w \in \mathbb{R}^{n}\right.$ : the minimum in $\operatorname{trop}(f)(w)$ is achieved at least twice $\}$.

Note that $\operatorname{trop}\left(x^{u} f\right)=\operatorname{trop}\left(x^{u}\right)+\operatorname{trop}(f), \operatorname{so} \operatorname{trop}\left(x^{u} f\right)(w)=w \cdot u+\operatorname{trop}(f)(w)$, and thus $\operatorname{trop}\left(V\left(x^{u} f\right)\right)=\operatorname{trop}(V(f))$. This explains why the natural algebraic varieties to tropicalize are subvarieties of $T=\left(K^{*}\right)^{n}$, rather than subvarieties of $\mathbb{A}^{n}$ or $\mathbb{P}^{n}$, as monomial functions are invertible on $T$. 


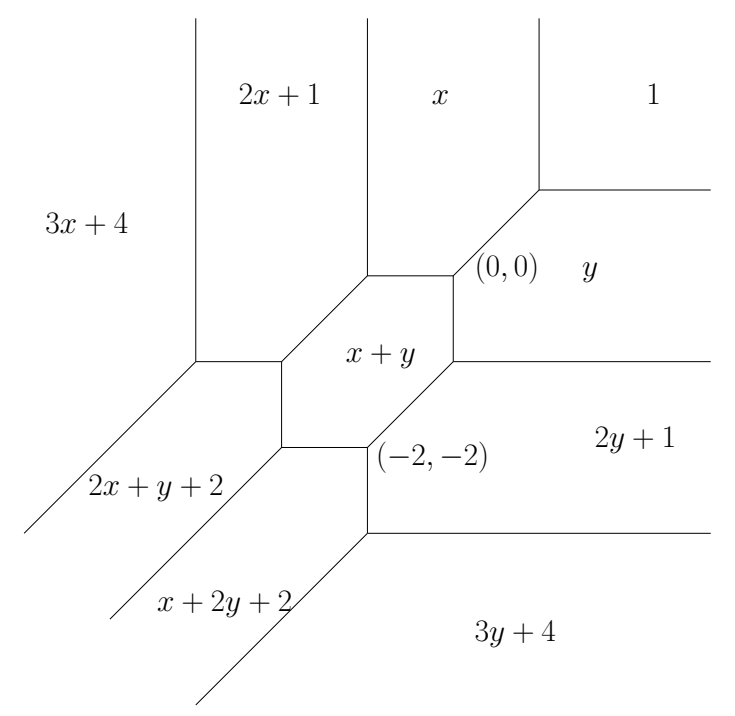

Figure 5. A tropical elliptic curve

Let $Y=V(I)$ be a subvariety of $T=\left(K^{*}\right)^{n}$. If $I=\left\langle f_{1}, \ldots, f_{r}\right\rangle$ then

$$
\begin{aligned}
Y=V(I) & =\left\{x \in T: f_{1}(x)=\cdots=f_{r}(x)=0\right\} \\
& =\{x \in T: f(x)=0 \text { for all } f \in I\} \\
& =\bigcap_{f \in I} V(f) .
\end{aligned}
$$

Definition 2.4. The tropicalization of a variety $Y=V(I) \subseteq T$ is

$$
\operatorname{trop}(Y)=\bigcap_{f \in I} \operatorname{trop}(V(f)) .
$$

EXAmple 2.5. (1) $Y=V(x+y+z+w, x+2 y+5 z+11 w) \subseteq\left(\mathbb{C}^{*}\right)^{4}$. Then $\operatorname{trop}(Y)$ has the property that if $\alpha \in \operatorname{trop}(Y)$, then $\alpha+\lambda(1,1,1,1) \in$ $\operatorname{trop}(Y)$ for all $\lambda \in \mathbb{R}$. We can thus quotient by the span of $(1,1,1,1)$ and describe $\operatorname{trop}(Y)$ in $\mathbb{R}^{4} / \mathbb{R}(1,1,1,1) \cong \mathbb{R}^{3}$. This consists of four rays, being the images of the positive coordinate directions $(1,0,0,0),(0,1,0,0)$, $(0,0,1,0)$, and $(0,0,0,1)$.

(2) Let $Y=V\left(t^{3} x^{3}+x^{2} y+x y^{2}+t^{3} y^{3}+x^{2}+t^{-1} x y+y^{2}+x+y+t^{3}\right) \subseteq\left(\mathbb{C}\{\{t\}\}^{*}\right)^{2}$. Then $\operatorname{trop}(V(f))$ is shown in Figure 5 This is a "tropical elliptic curve"; see, for example, [12] or [26].

REMARK 2.6. If $Y=V\left(f_{1}, \ldots, f_{r}\right)$, the tropical variety $\operatorname{trop}(Y)$ does not always equal $\bigcap_{i=1}^{r} \operatorname{trop}\left(V\left(f_{i}\right)\right)$. For example, in the first part of Example 2.5, $\operatorname{trop}(V(x+$ $y+z+w))=\operatorname{trop}(V(x+2 y+5 z+11 w))$, but this is larger than the tropical variety $\operatorname{trop}(Y)$. 
Definition 2.7. If $Y=V(I)$, a set $\left\{f_{1}, \ldots, f_{r}\right\} \subseteq I$ with

$$
\operatorname{trop}(Y)=\bigcap_{i=1}^{r} \operatorname{trop}\left(V\left(f_{i}\right)\right)
$$

is called a tropical basis for I. Finite tropical bases always exist (see [5] Theorem $11])$, so $\operatorname{trop}(Y)$ is a piecewise linear object.

The following is the guiding question behind much tropical geometry research:

QUESTION 2.8. Which properties of $Y$ or of compactifications of $Y$ can be deduced from $\operatorname{trop}(Y)$ ?

\section{Drawing curves in the plane}

Before delving deeper into the connections between classical and tropical varieties, with a view to partial answers to Question 2.8 we first describe how to draw plane curves.

Let $C=V(f) \subseteq\left(K^{*}\right)^{2}$ for $f \in K\left[x^{ \pm 1}, y^{ \pm 1}\right]$. If $f=\sum c_{i j} x^{i} y^{j}$, then $\operatorname{trop}(f)=$ $\min \left(\operatorname{val}\left(c_{i j}\right)+i x+j y\right)$, and $\operatorname{trop}(C)=\operatorname{trop}(V(f))$ is the locus where the minimum is achieved at least twice. Some elementary polyhedral geometry gives a shortcut to compute this locus, so we first review this notation. An excellent general introduction to polyhedral geometry is $\mathbf{3 9}$.

Definition 3.1. A polyhedron in $\mathbb{R}^{n}$ is the intersection of finitely many halfspaces in $\mathbb{R}^{n}$. This can be written as:

$$
P=\left\{x \in \mathbb{R}^{n}: A x \leq b\right\},
$$

where $A$ is a $d \times n$ matrix, and $b \in \mathbb{R}^{d}$. The dimension of $P$ is the dimension of the subspace $\operatorname{ker}(A)$. For a subgroup $\Gamma \subseteq \mathbb{R}$, we say that a polyhedron $P$ is $\Gamma$-rational if $A$ has rational entries, and $b \in \Gamma^{d}$. When $\Gamma=\mathbb{Q}$, we say that the polyhedron is rational.

If $b=0$, then $P$ is called a cone. In that case there are $\mathbf{v}_{1}, \ldots, \mathbf{v}_{s}$ for which $P=\operatorname{pos}\left(\mathbf{v}_{1}, \ldots, \mathbf{v}_{s}\right):=\left\{\sum_{i=1}^{s} \lambda_{i} \mathbf{v}_{i}: \lambda_{i} \geq 0\right\}$.

The face of a polyhedron $P$ determined by $w \in\left(\mathbb{R}^{n}\right)^{*}$ is the set

$$
\text { face }_{w}(P)=\{x \in P: w \cdot x \leq w \cdot y \text { for all } y \in P\} .
$$

EXAmPle 3.2. Let $P \subset \mathbb{R}^{2}$ be the square with vertices $\{(0,0),(1,0),(0,1),(1,1)\}$. This has the description

$$
P=\left\{x \in \mathbb{R}^{2}:\left(\begin{array}{rr}
1 & 0 \\
0 & 1 \\
-1 & 0 \\
0 & -1
\end{array}\right) x \leq\left(\begin{array}{l}
1 \\
1 \\
0 \\
0
\end{array}\right)\right\} .
$$

Then we have

(1) face $_{(1,0)}(P)$ is the edge of the square with vertices $\{(0,0),(0,1)\}$,

(2) face $_{(1,1)}(P)$ is the vertex $(0,0)$, and

(3) face $_{(0,0)}(P)$ is $P$.

Definition 3.3. A polyhedral complex $\Sigma$ is a finite union of polyhedra for which any nonempty intersection of two polyhedra $\sigma_{1}, \sigma_{2} \in \Sigma$ is a face of each. If every polyhedron in $\Sigma$ is a cone, then $\Sigma$ is called a fan. 
The normal fan to a polyhedron $P$ is the fan $N(P)$ with cones $C[w]=\operatorname{cl}\left(w^{\prime}\right.$ : face $_{w^{\prime}}(P)=$ face $_{w}(P)$ ), where $\operatorname{cl}(\cdot)$ is the closure in the usual Euclidean topology on $\mathbb{R}^{n}$. This is sometimes called the inner normal fan, as the cones corresponding to facets (faces of maximal dimension) are the inner normal vectors to these faces

Definition 3.4. Fix $f \in K\left[x_{1}^{ \pm 1}, \ldots, x_{n}^{ \pm 1}\right]$. The Newton polytope of $f=\sum c_{u} x^{u}$ is the convex hull of the $u \in \mathbb{Z}^{n}$ with $c_{u} \neq 0: P=\left\{\sum_{c_{u} \neq 0} \lambda_{u} u: \sum \lambda_{u}=1\right\}$.

ExAmPLE 3.5. Let $f=x^{2} y+5 y^{2}-3 x+2$. The Newton polytope $P$ of $f$ and its normal fan $N(P)$ are illustrated in Figure 6. The polytope $P$ is the convex hull of the exponent vectors $\{(2,1),(0,2),(1,0),(0,0)\}$.
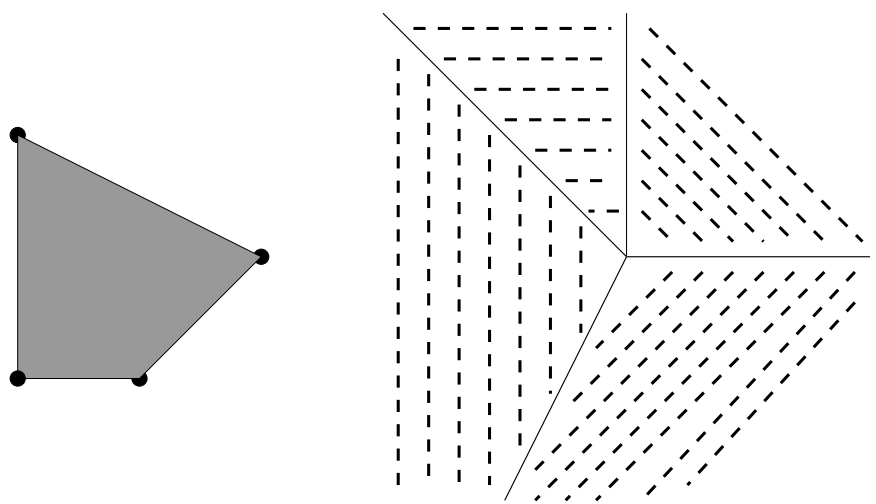

FIGURE 6. Newton polytope and normal fan

We now temporarily restrict our attention to plane curves, so $n=2$. The tropicalization of a plane curve $C=V(f)$ for $f=\sum c_{i j} x^{i} y^{j}$ depends on the valuations of the coefficients $c_{i j}$. If the valuation on $K$ is trivial $(\operatorname{val}(a)=0$ for all $a \neq 0$ ), then $\operatorname{trop}(C)$ is the union of all one-dimensional cones in the normal fan $N(P)$ to the Newton polytope of $f$.

We now consider the case that $K$ has a nontrivial valuation. Let $\widetilde{P}$ be the convex hull of the set $\left\{\left(i, j, \operatorname{val}\left(c_{i j}\right)\right): c_{i j} \neq 0\right\}$ in $\mathbb{R}^{2+1}=\mathbb{R}^{3}$, and let $N(\widetilde{P})$ be its normal fan. The regular subdivision $\Delta_{\left(\operatorname{val}\left(c_{i j}\right)\right)}$ of $P$ corresponding to the vector $\left(\operatorname{val}\left(c_{i j}\right)\right)$ is the projection to $P$ of the "lower faces" of $\widetilde{P}$. More information about regular subdivisions can be found in [20, Chapter 7] and [11, Chapter 2].

ExAMPLE 3.6. Let $f=2 x^{2}+x y-6 y^{2}+5 x-3 y+2 \in \mathbb{Q}\left[x^{ \pm 1}, y^{ \pm 1}\right]$ where $\mathbb{Q}$ has the 2-adic valuation. Then the regular subdivision of the Newton polytope of $f$ corresponding to $\operatorname{val}\left(c_{u}\right)$ is shown in Figure 7.

The tropicalization of $C$ is $\operatorname{trop}(C)=\left\{w \in \mathbb{R}^{2}: \operatorname{face}_{(w, 1)}(\widetilde{P})\right.$ is not a vertex $\}$. This is the reflection of the dual graph to $\Delta_{\left(\operatorname{val}\left(c_{i j}\right)\right)}$ under $x \mapsto-x$.

EXAMPLE 3.7. Let $f=27 x^{3}+6 x^{2} y+12 x y^{2}+81 y^{2}+3 x^{2}+5 x y+3 y^{2}+3 x+$ $2 y+243 \in \mathbb{Q}\left[x^{ \pm 1}, y^{ \pm 1}\right]$ where $\mathbb{Q}$ has the 3 -adic valuation.

Then the regular triangulation corresponding to $\left(\operatorname{val}\left(c_{i j}\right)\right)$ is shown in Figure 8 along with the tropical variety. 


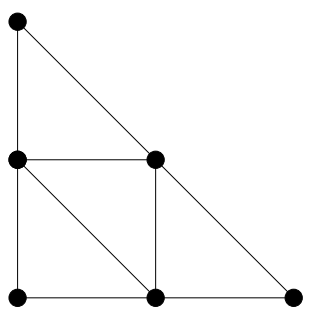

FiguRE 7 . The regular subdivision induced by $\left(\operatorname{val}\left(c_{u}\right)\right)$
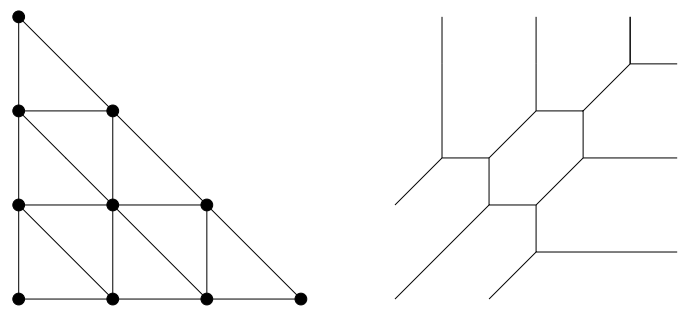

Figure 8. A tropical plane cubic

\section{The Fundamental and Structure theorems}

The definition of the tropical variety in Definition 2.4 was by analogy with the classical case. For an ideal $I$ the tropical variety $\operatorname{trop}(V(I))$ is the set of common "tropical zeros" of the tropicalizations of the polynomials $f \in I$. The connection between the tropicalization $\operatorname{trop}(Y)$ of a variety $Y=V(I) \subseteq\left(K^{*}\right)^{n}$ and the original variety $Y$ is closer than this analogy might suggest, as the following theorem shows.

THEOREM 4.1 (Fundamental theorem of tropical algebraic geometry). Let $K$ be an algebraically closed field with a nontrivial valuation val : $K^{*} \rightarrow \mathbb{R}$, and let $Y$ be a subvariety of $\left(K^{*}\right)^{n}$. Then

$$
\begin{aligned}
\operatorname{trop}(Y) & =\operatorname{cl}(\operatorname{val}(Y)) \\
& =\operatorname{cl}\left(\left(\operatorname{val}\left(y_{1}\right), \ldots, \operatorname{val}\left(y_{n}\right)\right): y=\left(y_{1}, \ldots, y_{n}\right) \in Y\right),
\end{aligned}
$$

where the closure is in the usual Euclidean topology on $\mathbb{R}^{n}$.

ExAmple 4.2. Let $Y=V(x+y+1) \subseteq\left(K^{*}\right)^{2}$, where $K=\mathbb{C}\{\{t\}\}$. Then $Y=\left\{(a,-1-a): a \in K^{*} \backslash\{-1\}\right\}$. Note that

$$
(\operatorname{val}(a), \operatorname{val}(-1-a))=\left\{\begin{array}{ll}
(\operatorname{val}(a), 0) & \text { if } \operatorname{val}(a)>0 \\
(\operatorname{val}(a), \operatorname{val}(a)) & \text { if } \operatorname{val}(a)<0, \\
(0, \operatorname{val}(b)) & \text { if } a=-1+b, \operatorname{val}(b)>0, \\
(0,0) & \text { otherwise. }
\end{array} .\right.
$$

Note that, as predicted by Theorem 4.1 the union of these sets is precisely $\operatorname{trop}(Y)$, as calculated in part 2 of Example 1.2. This is illustrated in Figure 9 which should be compared with Figure 2 . 


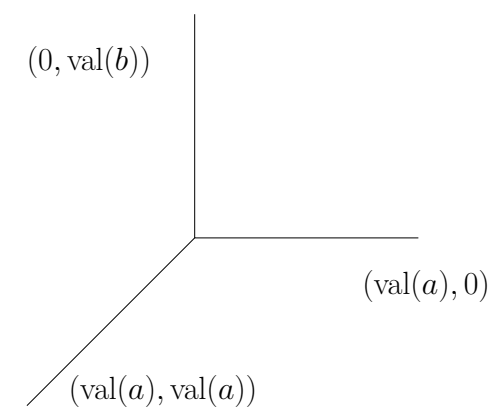

Figure 9. The Fundamental Theorem applied to a tropical line

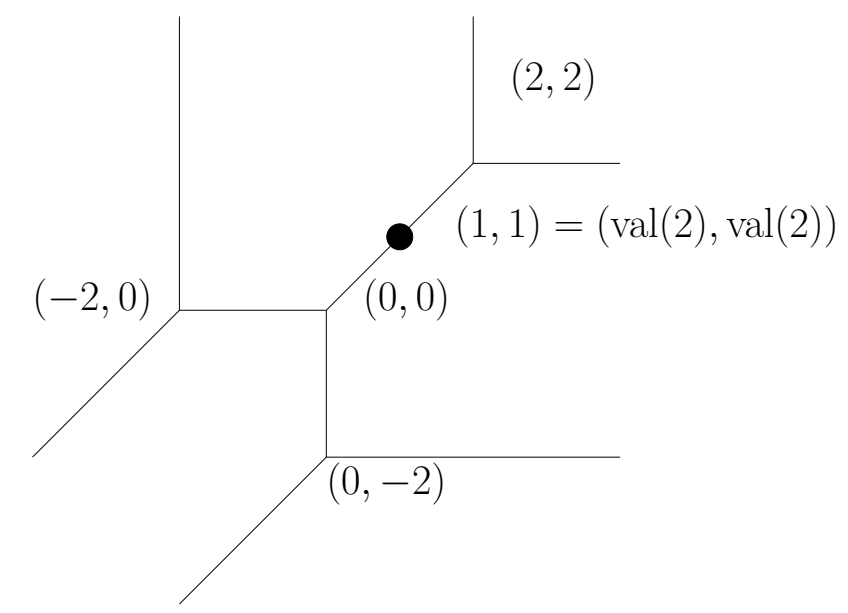

Figure 10. An example of the Fundamental Theorem

EXAmPle 4.3. Let $f=4 x^{2}+x y-4 y^{2}+x-y-4 \in \mathbb{Q}[x, y]$, where $\mathbb{Q}$ has the 2-adic valuation. Then $\operatorname{trop}(V(f))$ is shown in Figure 10, Note that the point $(2,2) \in V(f)$, and $(\operatorname{val}(2), \operatorname{val}(2))=(1,1) \in \operatorname{trop}(V(f))$.

Theorem 4.1 was first shown for hypersurfaces by Kapranov; see 14. The general case first appears in work of Speyer and Sturmfels [33, [34] see also the work of Draisma 13, Payne 32, and Jensen, Markwig, and Markwig [24. The hard part of Theorem 4.1 is to show that if $w \in \operatorname{trop}(Y) \cap(\mathrm{im} \text { val })^{n}$ then there is $y \in Y$ with $\operatorname{val}(y)=w$. Showing that $\{\operatorname{val}(y): y \in Y\} \subseteq \operatorname{trop}(Y)$ is comparatively easy.

The slogan form of Theorem 4.1 is then:

Tropical varieties are combinatorial shadows of classical varieties.

The word "combinatorial" is justified by the Structure Theorem for tropical varieties, which gives combinatorial constraints on which sets can be tropical varieties. The statement of this theorem requires some more polyhedral definitions.

Definition 4.4. Let $\Sigma$ be a polyhedral complex. The support of $\Sigma$ is the set

$$
|\Sigma|=\left\{x \in \mathbb{R}^{n}: x \in \sigma \text { for some } \sigma \in \Sigma\right\}
$$



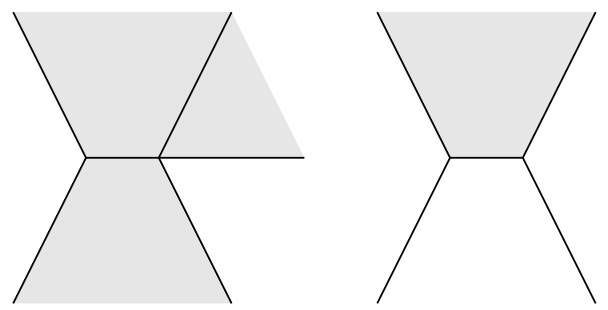

Figure 11. The complex on the left is pure, while the one on the right is not.

A polyhedral complex is pure if the dimension of every maximal polyhedron is the same. See Figure 11.

The lineality space $L$ of a polyhedral complex $\Sigma$ is the largest subspace of $\mathbb{R}^{n}$ for which $x+l \in \Sigma$ for all $x \in \Sigma, l \in L$. In part 1 of Example 2.5 the lineality space of $\operatorname{trop}(Y)$ is $\operatorname{span}((1,1,1,1)) \subseteq \mathbb{R}^{4}$.

Definition 4.5. A weighted polyhedral complex is a pure polyhedral complex $\Sigma$ with a weight $w_{\sigma} \in \mathbb{N}$ for all maximal-dimensional $\sigma \in \Sigma$.

Let $\Sigma$ be a weighted (im val)-rational polyhedral complex that is pure of dimension $d$. The complex $\Sigma$ is balanced if the following "zero-tension" conditions hold.

(1) If $\Sigma$ is a one-dimensional rational fan, let $\mathbf{u}_{1}, \ldots, \mathbf{u}_{s}$ be the first lattice points on the rays of $\Sigma$, and let $w_{i}$ be the weight of the cone containing the lattice point $\mathbf{u}_{i}$. Then $\Sigma$ is balanced if $\sum_{i=1}^{s} w_{i} \mathbf{u}_{i}=0$.

(2) For a general polyhedral complex $\Sigma$, fix a $(d-1)$-dimensional polyhedron $\tau$ of $\Sigma$. Let $L=\operatorname{span}(x-y: x, y \in \tau)$ be the affine span of $\tau$. Let $\operatorname{star}_{\Sigma}(\tau)$ be the rational polyhedral fan whose support is $\left\{w \in \mathbb{R}^{n}\right.$ : there exists $\epsilon>$ 0 for which $w^{\prime}+\epsilon w \in \Sigma$ for all $\left.w^{\prime} \in \tau\right\}+L$. This has one cone for each polyhedron $\sigma \in \Sigma$ that contains $\tau$, and has lineality space $L$. The quotient $\operatorname{star}_{\Sigma}(\tau) / L$ is a one dimensional fan which inherits weights from $\Sigma$. We say that $\Sigma$ is balanced at $\tau$ if the one-dimensional fan $\operatorname{star}_{\Sigma}(\tau) / L$ is balanced. The polyhedral complex $\Sigma$ is balanced if $\Sigma$ is balanced at all $(d-1)$ dimensional cones.

EXAmple 4.6. Let $f=x^{2} y^{2}+x^{3}+y^{3}+1 \in \mathbb{C}\left[x^{ \pm 1}, y^{ \pm 1}\right]$. Then $\operatorname{trop}(V(f))$ is a one-dimensional fan with four rays: $\operatorname{pos}((1,0)), \operatorname{pos}((0,1)), \operatorname{pos}((-2,-1))$, and $\operatorname{pos}((-1,-2))$. This is balanced with weights $3,3,1$, and $1: 3(1,0)+3(0,1)+$ $1(-2,-1)+1(-1-2)=(0,0)$.

Definition 4.7. We associate a graph to a pure polyhedral complex $\Sigma$, with a vertex for each maximal polyhedron $\sigma \in \Sigma$, and an edge between two vertices if the corresponding polyhedra intersect in a codimension-one face. The polyhedral complex $\Sigma$ is connected through codimension-one if this graph is connected. For example, the polyhedral complex on the left of Figure 12 is connected through codimension-one, while the one on the right is not.

Recall that a variety $Y \subseteq\left(K^{*}\right)^{n}$ is irreducible if we cannot write $Y=Y_{1} \cup Y_{2}$ for $Y_{1}, Y_{2}$ nonempty proper subvarieties of $Y$. Note that by Theorem 4.1 we have $\operatorname{trop}\left(Y_{1} \cup Y_{2}\right)=\operatorname{trop}\left(Y_{1}\right) \cup \operatorname{trop}\left(Y_{2}\right)$. 


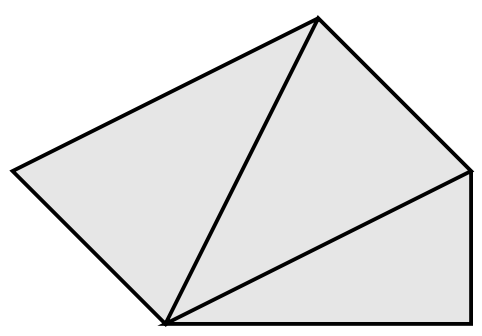

Connected through codimension one

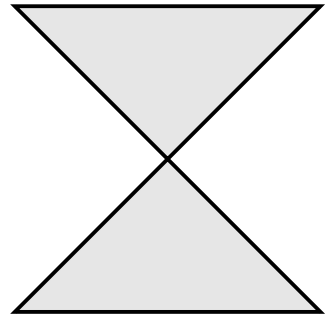

Not connected through codimension one

FIGURE 12. A polyhedral complex that is (left) and is not (right) connected through codimension one

The following "Structure Theorem" summarizes the combinatorial structure on the tropical variety. This is essentially due to Bieri and Groves [3], with the stronger connectedness statement from [5] and [7].

ThEOREM 4.8 (Structure Theorem). Let $Y$ be a d-dimensional irreducible subvariety of $\left(K^{*}\right)^{n}$. Then $\operatorname{trop}(Y)$ is the support of a pure d-dimensional weighted balanced (im val)-rational polyhedral complex that is connected through codimensionone.

The Structure Theorem means that the tropical variety has a discrete structure, and records information about the original variety (such as its dimension).

The weights $w_{\sigma}$ on maximal cones that make trop $(Y)$ balanced can be computed from $Y$, as we explain in the next section.

\section{The computational approach to tropical varieties}

An important aspect of tropical varieties is that they can actually be computed in practice. This uses an extension of the theory of Gröbner bases to fields with a valuation.

Fix a splitting (im val) $\rightarrow K^{*}$ of the valuation. This is a group homomorphism $u \mapsto t^{u}$ with $\operatorname{val}\left(t^{u}\right)=u$. For example, when $K=\mathbb{C}$ with the trivial valuation $(\operatorname{val}(a)=0$ for all $a \neq 0)$, then we can choose the splitting $0 \mapsto 1$. When $K=$ $\mathbb{C}\{\{t\}\}$, we can choose $u \mapsto t^{u}$, and when $K=\mathbb{Q}$ with the $p$-adic valuation, we can choose $u \mapsto p^{u}$. Such splittings always exist when $K$ is algebraically closed; see [28, Lemma 2.1.13].

Let $R=\{a \in K: \operatorname{val}(a) \geq 0\}$ be the valuation $\operatorname{ring}$ of $K$. The ring $R$ is local, with maximal ideal $\mathfrak{m}=\{a \in K: \operatorname{val}(a)>0\} \cup\{0\}$. The quotient $\mathbb{k}=R / \mathfrak{m}$ is the residue field.

ExAmPle 5.1. (1) When $K=\mathbb{C}$ has the trivial valuation, we have $R=$ $\mathbb{C}$, and $\mathfrak{m}=0$, so $\mathbb{k}=\mathbb{C}$.

(2) When $K=\mathbb{C}\{\{t\}\}, R=\bigcup_{n \geq 1} \mathbb{C} \llbracket t^{1 / n} \rrbracket$, and $\mathbb{k}=\mathbb{C}$.

(3) When $K=\mathbb{Q}_{p}, R=\mathbb{Z}_{p}$, and $\mathbb{k}=\mathbb{Z} / p \mathbb{Z}$.

Given a polynomial $f=\sum c_{u} x^{u} \in K\left[x_{1}^{ \pm 1}, \ldots, x_{n}^{ \pm 1}\right]$, and $w \in(\text { im val })^{n}$, the initial form is

$$
\operatorname{in}_{w}(f)=\sum_{\operatorname{val}\left(c_{u}\right)+w \cdot u=\operatorname{trop}(f)(w)} \overline{t^{-\operatorname{val}\left(c_{u}\right)} c_{u}} x^{u} \in \mathbb{k}\left[x_{1}^{ \pm 1}, \ldots, x_{n}^{ \pm 1}\right],
$$




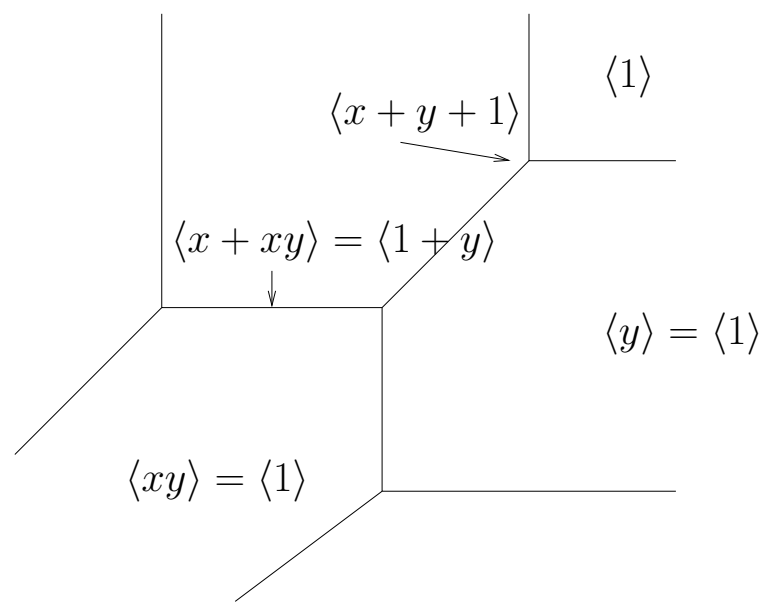

Figure 13. The Gröbner complex of Example 5.4

where for $a \in R$ we denote by $\bar{a}$ the image of $a$ in $\mathbb{k}$.

ExAmple 5.2. Let $K=\mathbb{Q}$ with the 2 -adic valuation, and let $f=2 x^{2}+x y+$ $6 y^{2}+5 x-3 y+4$. Then for $w=(2,2)$ we have $\operatorname{trop}(f)(w)=2$, so $\operatorname{in}_{w}(f)=$ $\overline{5} x+\overline{-3} y+\overline{2^{-2} 4}=x+y+1 \in \mathbb{Z} / 2 \mathbb{Z}\left[x^{ \pm 1}, y^{ \pm 1}\right]$.

For $w=(-2,-1)$ we have $\operatorname{trop}(f)(w)=-3$, $\operatorname{so~in}_{w}(f)=x^{2}+x y$.

Given an ideal $I \subset K\left[x_{1}^{ \pm 1}, \ldots, x_{n}^{ \pm 1}\right]$ and $w \in(\mathrm{im} v a l)^{n}$, the initial ideal of $I$ is

$$
\operatorname{in}_{w}(I)=\left\langle\operatorname{in}_{w}(f): f \in I\right\rangle .
$$

This is a variant of the standard theory of Gröbner bases for ideals in a polynomial ring; see 8 for an excellent introduction to the standard case. As in that setting, the initial ideal need not be generated by the initial forms of a generating set for $I$. There are always finite generating sets for $I$, however, for which this is the case. These finite sets (Gröbner bases) can be computed using a variant of the standard Gröbner basis algorithm.

The connection of this theory of Gröbner bases to tropical geometry is the following computational characterization of tropical varieties, due to Sturmfels $\mathbf{3 6}$. Chapter 9].

Proposition 5.3. Let $Y=V(I) \subseteq\left(K^{*}\right)^{n}$ and let $w \in(\mathrm{imval})^{n}$. Then $w \in$ $\operatorname{trop}(Y)$ if and only if $\operatorname{in}_{w}(I) \neq\langle 1\rangle \subseteq \mathbb{k}\left[x_{1}^{ \pm 1}, \ldots, x_{n}^{ \pm 1}\right]$.

Example 5.4. Let $I=\left\langle 2 x^{2}+x y+6 y^{2}+5 x-3 y+4\right\rangle \in \mathbb{Q}\left[x^{ \pm 1}, y^{ \pm 1}\right]$, where $\mathbb{Q}$ has the 2-adic valuation. The claim of Proposition 5.3 is illustrated in Figure 13 ,

There is a polyhedral complex $\Sigma$ with $\operatorname{in}_{w}(I)$ constant for $w \in \operatorname{relint}(\sigma)$ for any $\sigma \in \Sigma$. This is the Gröbner complex of a homogenization of $I$. In the case that $K=\mathbb{C}$ with the trivial valuation the Gröbner complex is a polyhedral fan, called the Gröbner fan. See [2], 31, [35. Chapter 2] for more on the Gröbner fan, and 27, 28 for expositions of the Gröbner complex.

Proposition 5.3 implies that $\operatorname{trop}(Y)$ is the union of the polyhedra $\sigma$ in the Gröbner complex of $I(Y)$ for which $\operatorname{in}_{w}(I) \neq\langle 1\rangle$ for any $w \in \operatorname{relint}(\sigma)$. The 


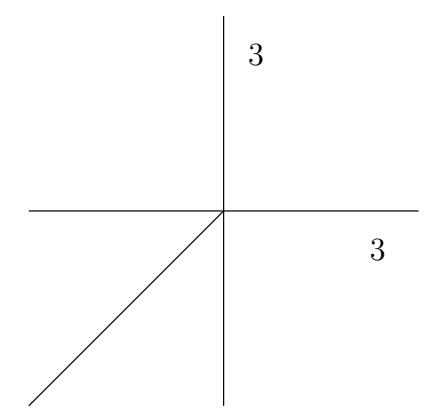

Figure 14. The balancing condition

software gfan 23 by Anders Jensen computes tropical varieties by exploiting this Gröbner description.

The Structure Theorem (Theorem 4.8) asserts that $\operatorname{trop}(Y)$ is the support of a weighted balanced polyhedral complex. The weights that make the polyhedral complex balanced can be defined using the Gröbner theory we have just summarized. We assume that the polyhedral complex structure $\Sigma$ has been chosen, as described above, so that $\operatorname{in}_{w}(I(Y))$ is constant on the relative interior of polyhedra of $\Sigma$. Fix $w \in(\mathrm{imval})^{n}$ in the relative interior of a maximal polyhedron of $\Sigma$. Then $V\left(\operatorname{in}_{w}(I(Y))\right) \subseteq\left(\mathbb{k}^{*}\right)^{n}$ is a union of $\left(\mathbb{k}^{*}\right)^{d}$-orbits, where $d=\operatorname{dim}(Y)$ (see [28, Chapter 3] for details). We set $w_{\sigma}$ to be the number of such orbits (counted with multiplicity).

Hidden in the proof of the Structure Theorem is the fact that this choice makes the polyhedral complex $\Sigma$ balanced.

EXAmple 5.5. Let $f=x^{2}+3 x+2+x^{2} y+2 x y^{2}-2 y^{2} \subseteq \mathbb{C}\left[x^{ \pm 1}, y^{ \pm 1}\right]$. Then $\operatorname{trop}(V(f))$ is a one-dimensional fan with five rays, spanned by the vectors $\{(1,0),(0,1),(-1,0),(-1,-1),(0,-1)\}$. When $w=(0,1), \operatorname{trop}(f)(w)=0$, so $\operatorname{in}_{w}(f)=x^{2}+3 x+2=(x+2)(x+1)$. We then have $V\left(\operatorname{in}_{w}(f)\right)=\{(-2, a)$ : $\left.a \in \mathbb{C}^{*}\right\} \cup\left\{(-1, a): a \in \mathbb{C}^{*}\right\}$. The weight on the cone spanned by $(0,1)$ is thus 2 . When $w=(-1,-1), \operatorname{in}_{w}(f)=x^{2} y+2 x y^{2}$, so $V\left(\operatorname{in}_{w}(f)\right)=V(x+2 y)=\{(2 a,-a)$ : $\left.a \in \mathbb{C}^{*}\right\}$. This makes the weight on this cone 1 . Similarly, the weight on the cone spanned by $(1,0)$ is 2 and all other weights are 1 . Note that

$$
2\left(\begin{array}{l}
1 \\
0
\end{array}\right)+2\left(\begin{array}{l}
0 \\
1
\end{array}\right)+1\left(\begin{array}{r}
-1 \\
0
\end{array}\right)+1\left(\begin{array}{l}
-1 \\
-1
\end{array}\right)+1\left(\begin{array}{r}
0 \\
-1
\end{array}\right)=\left(\begin{array}{l}
0 \\
0
\end{array}\right) .
$$

This is illustrated in Figure 14

\section{Connections to subvarieties of toric varieties}

Tropical varieties record information not just about subvarieties of the algebraic torus $\left(K^{*}\right)^{n}$, but also about their compactifications. This vastly extends the reach of tropical techniques; for example, every projective variety is a compactification of a subvariety of the algebraic torus. The connection is through the theory of toric varieties.

We assume here that $K=\mathbb{C}$. This means that for $Y \in\left(\mathbb{C}^{*}\right)^{n}$, $\operatorname{trop}(Y)$ is the support of a weighted balanced rational polyhedral fan. 
Definition 6.1. A (normal) toric variety is a normal variety $X$ containing a dense copy of $T=\left(\mathbb{C}^{*}\right)^{n}$ with an action of $T$ on $X$ that extends the action of $T$ on itself.

Examples include:

(1) $X=\left(\mathbb{C}^{*}\right)^{n}$,

(2) $X=\mathbb{A}^{n} \supset\left(\mathbb{C}^{*}\right)^{n}=\left\{x \in \mathbb{A}^{n}: x_{i} \neq 0\right.$ for $\left.1 \leq i \leq n\right\}$,

(3) $X=\mathbb{P}^{n} \supset\left(\mathbb{C}^{*}\right)^{n}=\left\{x \in \mathbb{P}^{n}: x_{i} \neq 0\right.$ for $\left.0 \leq i \leq n\right\}$,

(4) $X=\mathbb{P}^{1} \times \mathbb{P}^{1} \supset\left(\mathbb{C}^{*}\right)^{2}$.

A toric variety $X$ is a union of $T$-orbits. These can be recorded using a polyhedral fan $\Sigma$.

EXAmple 6.2 . The projective plane $\mathbb{P}^{2}$ decomposes into the following $T=$ $\left(\mathbb{C}^{*}\right)^{2}$-orbits:

$$
\begin{gathered}
\left(\mathbb{C}^{*}\right)^{2} \cup\left\{[0: a: b]: a, b \in \mathbb{C}^{*}\right\} \cup\left\{[a: 0: b]: a, b \in \mathbb{C}^{*}\right\} \cup\left\{[a: b: 0]: a, b \in \mathbb{C}^{*}\right\} \\
\cup\{[1: 0: 0]\} \cup\{[0: 1: 0]\} \cup\{0: 0: 1]\} .
\end{gathered}
$$

The corresponding fan is shown in Figure 15 .

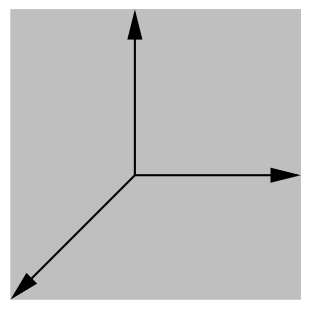

Figure 15 . The fan of $\mathbb{P}^{2}$

Alternatively (and more standardly), given a rational polyhedral fan $\Sigma$ we construct a toric variety $X_{\Sigma}$ by gluing together torus orbits closures. Each cone of $\Sigma$ determines an affine toric variety, and the fan tells us how to glue them together. For example, for $\mathbb{P}^{2}$ the fan tells us to construct $\mathbb{P}^{2}$ by gluing together the three affine charts $\left\{x \in \mathbb{P}^{2}: x_{i} \neq 0\right\}$ for $0 \leq i \leq 2$. For more background on toric varieties, some good references include $\mathbf{9}$ and $\mathbf{1 7}$.

The connection to tropical geometry begins with the following question.

Question 6.3. Given a toric variety $X_{\Sigma}$, and a subvariety $\bar{Y} \subseteq X_{\Sigma}$, which $T$-orbits of $X_{\Sigma}$ does $\bar{Y}$ intersect?

Surprisingly, the answer uses tropical geometry. The subvariety $\bar{Y}$ intersects the torus orbit indexed by $\sigma \in \Sigma$ if and only if $\operatorname{trop}(\bar{Y} \cap T)$ intersects $\operatorname{relint}(\sigma)$. This follows from work of Tevelev [38].

EXAmple 6.4. Let $\bar{Y}=V(x+y+z) \subseteq \mathbb{P}^{2}$. Then $\operatorname{trop}(\bar{Y} \cap T)=\operatorname{trop}(x+y+1)$, which is the standard tropical line. This intersects every one of the cones of the fan of $\mathbb{P}^{2}$ except for the top-dimensional ones. Indeed, the top-dimensional cones correspond to the $T$-fixed points $[1: 0: 0],[0: 1: 0]$ and $[0: 0: 1]$, which do not lie in $\bar{Y}$, while every other $T$-orbit does contain a point of $\bar{Y}$. 
A fundamental problem in algebraic geometry, especially in the consideration of moduli spaces, is to find a good compactification of a variety. Here the notion of "good" will depend on the specific problem, but we often require the compactification to be smooth, the "boundary" (new points added in the compactification) to be a divisor (codimension-one), and the irreducible components of this divisor to intersect nicely.

ExAmple 6.5. Let $\mathcal{A}=\left\{H_{1}, \ldots, H_{s}\right\}$ be a hyperplane arrangement in $\mathbb{P}^{n}$, where $H_{i}=\left\{x \in \mathbb{P}^{n}: a_{i} \cdot x=0\right\}$, with $a_{i} \in \mathbb{C}^{n+1}$. Let $Y=\mathbb{P}^{n} \backslash \mathcal{A}$. This can be embedded into $\left(\mathbb{C}^{*}\right)^{s-1}$ by sending $y \in Y$ to $\left[a_{1} \cdot y: \cdots: a_{s} \cdot y\right]$. Let $A$ be the $(n+1) \times s$ matrix with columns the vectors $a_{i}$, which we assume has rank $n+1$, and let $B$ be a $(s-n-1) \times(n+1)$ matrix of rank $s-n-1$ with $A B^{T}=0$. Then $Y=V\left(\sum_{i=1}^{n} b_{i j} x_{j}: 1 \leq i \leq s-n-1\right)$. One choice of compactification of $Y$ is the original $\mathbb{P}^{n}$; another is the DeConcini-Procesi wonderful compactification [10].

A natural way to compactify a variety is to take its closure in a larger variety. The idea we follow, due to Tevelev [38, is to take as the larger variety a toric variety whose fan has support the tropical variety. Note that if $\operatorname{dim}(Y)>1$ there is not a unique such fan, and there may be no preferred choice.

Definition 6.6. Fix $Y \subset T$, and choose a fan $\Sigma$ with support $\operatorname{trop}(Y)$. The closure $\bar{Y}=\operatorname{cl}\left(Y \subset X_{\Sigma}\right)$ is a tropical compactification of $Y$.

Tropical compactifications have nice properties:

(1) $\bar{Y}$ is proper (compact),

(2) $\bar{Y}$ intersects a codimension- $k T$-orbit of $X_{\Sigma}$ in codimension $k$.

The first condition is somewhat surprising, since the toric variety $X_{\Sigma}$ is not itself compact. For example, if $Y=V(x+y+1) \subseteq\left(\mathbb{C}^{*}\right)^{2}$, then $\operatorname{trop}(Y)$ is the standard tropical line. There is only one polyhedral fan $\Sigma$ with this support, and the corresponding toric variety is $\mathbb{P}^{2}$ with three points removed, which is not compact. However in this case the closure of $Y$ in $\mathbb{P}^{2}$, which is clearly compact, does not pass through these three points, so the closure of $Y$ in $X_{\Sigma}$ equals the closure of $Y$ in $\mathbb{P}^{2}$, so is also compact.

The second condition means that the pull-backs to $\bar{Y}$ of the codimension- $k$ torus-invariant strata on $X_{\Sigma}$ give a finite number of distinguished classes in the Chow group $A^{k}(\bar{Y})$. For example when $k=1$ this gives a distinguished collection of effective divisors on $\bar{Y}$.

If the fan $\Sigma$ is chosen to be sufficiently refined then we get further control over the compactification $\bar{Y}$. One way to guarantee "sufficiently refined" is to choose a fan $\Sigma$ so that $\operatorname{in}_{w}(I(Y))$ is constant for all $w \in \operatorname{relint} \sigma$ for all $\sigma \in \Sigma$. By further refining $\Sigma$ we can also assume that the toric variety $X_{\Sigma}$ is smooth. With a sufficiently refined fan we have:

(1) The multiplicity $w_{\sigma}$ on a maximal cone $\sigma$ of $\Sigma$ equals the intersection number $[\bar{Y}] \cdot[V(\sigma)]$, where $V(\sigma)$ is the closure of the $T$-orbit corresponding to $\sigma$.

(2) For any $\sigma \in \Sigma$, the intersection of $\bar{Y}$ with the $T$-orbit $\mathcal{O}_{\sigma}$ is the quotient $V\left(\operatorname{in}_{w}(I(Y))\right) /\left(\mathbb{C}^{*}\right)^{\operatorname{dim} \sigma}$ for any $w \in \operatorname{relint}(\sigma)$.

See also [22, 33], and [37] for more details on this. The connection to intersection theory has been further developed by Allermann and Rau [1, which is the tropical analogue of earlier toric work by Fulton and Sturmfels $\mathbf{1 8}$. 
ExAmple 6.7. When $Y=\mathbb{P}^{n} \backslash \mathcal{A}$, as in Example 6.5 there is a coarsest fan structure $\Sigma$ on $\operatorname{trop}(Y) \subseteq \mathbb{R}^{s-1}$, since every other fan with support $\operatorname{trop}(Y)$ has cones that subdivide those of $\Sigma$. The tropical compactification $\bar{Y}$ coming from taking the closure in $X_{\Sigma}$ is the DeConcini/Procesi wonderful compactification for most choices of $\mathcal{A}$; see $\mathbf{1 5}$ and $3 \mathbf{3 8}$.

A motivating example of this is given by the moduli space $\bar{M}_{0, n}$. The moduli space $M_{0, n}$ parameterizes isomorphism classes of smooth genus zero curves with $n$ distinct marked points. It thus parameterizes ways to arrange $n$ distinct point on $\mathbb{P}^{1}$ up to $\operatorname{Aut}\left(\mathbb{P}^{1}\right)$. For example, $M_{0,3}$ is a point, as there is an automorphism of $\mathbb{P}^{1}$ that takes any three distinct points to $0,1, \infty$. When $n=4, M_{0,4}=\mathbb{P}^{1} \backslash\{0,1, \infty\}$. In general,

$$
\begin{aligned}
M_{0, n} & =\left(\mathbb{P}^{1} \backslash\{0,1, \infty\}\right)^{n-3} \backslash \text { diagonals } \\
& =\left(\mathbb{C}^{*} \backslash\{1\}\right)^{n-3} \backslash \text { diagonals } \\
& =\mathbb{P}^{n-3} \backslash\left\{x_{0}=0, x_{i}=0, x_{i}=x_{0}, x_{i}=x_{j}: 1 \leq i<j \leq n\right\} .
\end{aligned}
$$

We thus have $M_{0, n}$ as the complement of $\left(\begin{array}{c}n-1 \\ 2\end{array}\right)=\left(\begin{array}{c}n \\ 2\end{array}\right)-n+1$ hyperplanes. This means the moduli space $M_{0, n}$ can be be embedded into $\left(\mathbb{C}^{*}\right)^{\left(\begin{array}{l}n \\ 2\end{array}\right)-n}$ as a closed subvariety as in Example 6.5. The tropical variety $\operatorname{trop}\left(M_{0, n}\right)$ is the support of a $(n-3)$-dimensional fan $\Delta$ in $\mathbb{R}^{\left(\begin{array}{l}n \\ 2\end{array}\right)-n}$. The toric variety $X_{\Delta}$ is smooth, but not complete (projective).

The fan $\Delta$ is the space of phylogenetic trees, which comes from mathematical biology. See, for example, 4 for more on this space, and 34 for some of the connection, which uses Kapranov's description 25. of $\bar{M}_{0, n}$ as the Chow quotient of a Grassmannian. Maximal cones of $\Delta$ are labelled by trivalent trees with $n$ labelled leaves. A point in the cone records the length of the internal edges in the tree.

A picture of $\Delta$ when $n=5$ is shown in Figure16. This is a two-dimensional fan in $\mathbb{R}^{5}$, so its intersection with the four-dimensional sphere in $\mathbb{R}^{5}$ is a graph, which is drawn in Figure 16 .

The closure of $M_{0, n}$ in $X_{\Delta}$ is the Deligne-Mumford moduli space $\bar{M}_{0, n}$ of stable genus zero curves with $n$ marked points. This parameterizes isomorphism classes of trees of $\mathbb{P}^{1}$ 's with $n$ marked points and at least three special points (nodes or marked points) on each component. See [21] or [38] for details.

For $\sigma \in \Delta$, the intersection of $\bar{M}_{0, n}$ with the torus orbit corresponding to $\sigma$ is the stratum of all curves with dual graph the corresponding tree. In particular, the intersection of $\bar{M}_{0, n}$ with a torus-invariant divisor on $X_{\Delta}$ is a boundary divisor.

The moduli space $\bar{M}_{0, n}$ and the toric variety $X_{\Delta}$ are closely related. Their Picard groups are isomorphic, and the inclusion $i: \bar{M}_{0, n} \rightarrow X_{\Delta}$ introduces an isomorphism $i^{*}: A^{*}\left(X_{\Delta}\right) \rightarrow A^{*}\left(\bar{M}_{0, n}\right)$. This generalizes to general wonderful compactifications; see [16.

\section{References}

1. Lars Allermann and Johannes Rau, First steps in tropical intersection theory, Math. Z. 264 (2010), no. 3, 633-670. 


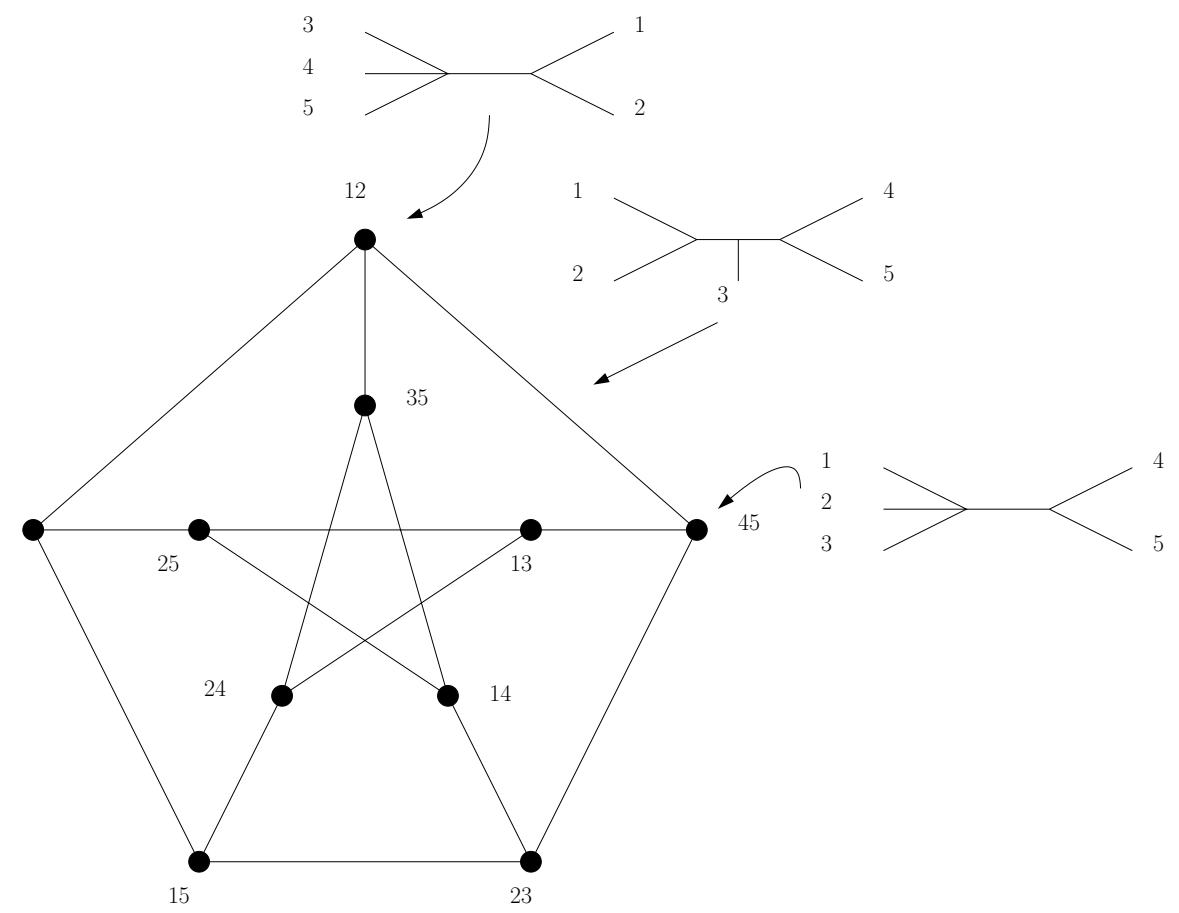

Figure 16. The tropical variety of $M_{0,5}$

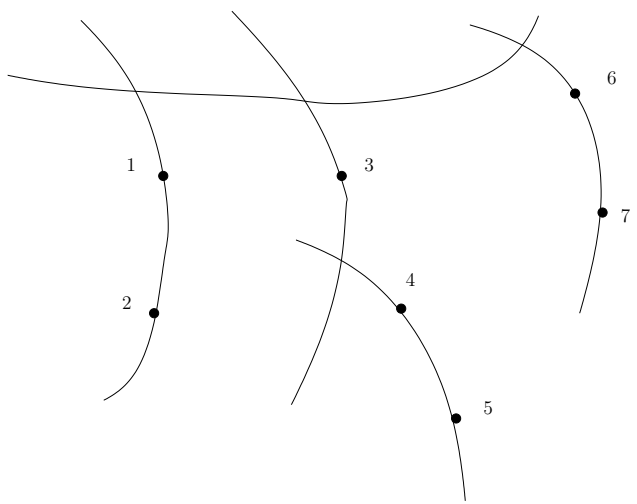

Figure 17. A stable curve with 7 marked points

2. David Bayer and Ian Morrison, Standard bases and geometric invariant theory. I. Initial ideals and state polytopes, J. Symbolic Comput. 6 (1988), no. 2-3, 209-217, Computational aspects of commutative algebra.

3. Robert Bieri and J. R. J. Groves, The geometry of the set of characters induced by valuations, J. Reine Angew. Math. 347 (1984), 168-195.

4. Louis J. Billera, Susan P. Holmes, and Karen Vogtmann, Geometry of the space of phylogenetic trees, Adv. in Appl. Math. 27 (2001), no. 4, 733-767.

5. Tristram Bogart, Anders N. Jensen, David Speyer, Bernd Sturmfels, and Rekha R. Thomas, Computing tropical varieties, J. Symbolic Comput. 42 (2007), no. 1-2, 54-73. 
6. Peter Butkovič, Max-linear systems: theory and algorithms, Springer Monographs in Mathematics, Springer-Verlag London Ltd., London, 2010.

7. Dustin Cartwright and Sam Payne, Connectivity of tropicalizations, arXiv:1204.6589, 2012.

8. David Cox, John Little, and Donal O'Shea, Ideals, varieties, and algorithms, third ed., Undergraduate Texts In Mathematics, Springer, New York, 2007, An Introduction To Computational Algebraic Geometry And Commutative Algebra.

9. David A. Cox, John B. Little, and Henry K. Schenck, Toric varieties, Graduate Studies in Mathematics, vol. 124, American Mathematical Society, Providence, RI, 2011.

10. Corrado De Concini and Claudio Procesi, Wonderful models of subspace arrangements, Selecta Math. (N.S.) 1 (1995), no. 3, 459-494.

11. Jesús A. De Loera, Jörg Rambau, and Francisco Santos, Triangulations, Algorithms and Computation in Mathematics, vol. 25, Springer-Verlag, Berlin, 2010, Structures for algorithms and applications.

12. Magnus Dehli Vigeland, The group law on a tropical elliptic curve, Math. Scand. 104 (2009), no. $2,188-204$.

13. Jan Draisma, A tropical approach to secant dimensions, J. Pure Appl. Algebra 212 (2008), no. $2,349-363$.

14. Manfred Einsiedler, Mikhail Kapranov, and Douglas Lind, Non-Archimedean amoebas and tropical varieties, J. Reine Angew. Math. 601 (2006), 139-157.

15. Eva Maria Feichtner and Bernd Sturmfels, Matroid polytopes, nested sets and Bergman fans, Port. Math. (N.S.) 62 (2005), no. 4, 437-468.

16. Eva Maria Feichtner and Sergey Yuzvinsky, Chow rings of toric varieties defined by atomic lattices, Invent. Math. 155 (2004), no. 3, 515-536.

17. William Fulton, Introduction to toric varieties, Annals of Mathematics Studies, vol. 131, Princeton University Press, Princeton, NJ, 1993, The William H. Roever Lectures in Geometry.

18. William Fulton and Bernd Sturmfels, Intersection theory on toric varieties, Topology 36 (1997), no. 2, 335-353.

19. Stéphane Gaubert and Max Plus, Methods and applications of $(\max ,+)$ linear algebra, STACS 97 (Lübeck), Lecture Notes in Comput. Sci., vol. 1200, Springer, Berlin, 1997, pp. 261-282.

20. Israel M. Gelfand, Mikhail M. Kapranov, and Andrei V. Zelevinsky, Discriminants, resultants and multidimensional determinants, Modern Birkhäuser Classics, Birkhäuser Boston Inc., Boston, MA, 2008, Reprint of the 1994 edition.

21. Angela Gibney and Diane Maclagan, Equations for Chow and Hilbert quotients, Algebra Number Theory 4 (2010), no. 7, 855-885.

22. Paul Hacking, The homology of tropical varieties, Collect. Math. 59 (2008), no. 3, 263-273.

23. Anders N. Jensen, Gfan, a software system for Gröbner fans and tropical varieties, Available at http://home.imf.au.dk/jensen/software/gfan/gfan.html.

24. Anders Nedergaard Jensen, Hannah Markwig, and Thomas Markwig, An algorithm for lifting points in a tropical variety, Collect. Math. 59 (2008), no. 2, 129-165.

25. Mikahil M. Kapranov, Chow quotients of Grassmannians. I, I. M. Gel'fand Seminar, Adv. Soviet Math., vol. 16, Amer. Math. Soc., Providence, RI, 1993, pp. 29-110.

26. Eric Katz, Hannah Markwig, and Thomas Markwig, The $j$-invariant of a plane tropical cubic, J. Algebra 320 (2008), no. 10, 3832-3848.

27. Diane Maclagan, Polyhedral structures on tropical varieties, Available at http://www.warwick.ac.uk/staff/D.Maclagan/papers/Bellairs.pdf. 2011.

28. Diane Maclagan and Bernd Sturmfels, Introduction to tropical geometry, Draft Book In Progress. Available at http://www.warwick.ac.uk/staff/D.Maclagan/papers/Tropicalbook.pdf

29. Grigory Mikhalkin, Introduction to tropical geometry (notes from the IMPA lectures), arXiv:0709.1049

30. Tropical geometry and its applications, International Congress of Mathematicians. Vol. II, Eur. Math. Soc., Zürich, 2006, pp. 827-852.

31. Teo Mora and Lorenzo Robbiano, The Gröbner fan of an ideal, J. Symbolic Comput. 6 (1988), no. 2-3, 183-208, Computational aspects of commutative algebra.

32. Sam Payne, Fibers of tropicalization, Math. Z. 262 (2009), no. 2, 301-311.

33. David Speyer, Tropical geometry, Ph.D. thesis, University of California, Berkeley, 2005. 
34. David Speyer and Bernd Sturmfels, The tropical Grassmannian, Adv. Geom. 4 (2004), no. 3, 389-411.

35. Bernd Sturmfels, Gröbner bases and convex polytopes, University Lecture Series, vol. 8, American Mathematical Society, Providence, RI, 1996.

36. Solving systems of polynomial equations, CBMS Regional Conference Series in Mathematics, vol. 97, Published for the Conference Board of the Mathematical Sciences, Washington, DC, 2002.

37. Bernd Sturmfels and Jenia Tevelev, Elimination theory for tropical varieties, Math. Res. Lett. 15 (2008), no. 3, 543-562.

38. Jenia Tevelev, Compactifications of subvarieties of tori, Amer. J. Math. 129 (2007), no. 4, $1087-1104$

39. Günter M. Ziegler, Lectures on polytopes, Graduate Texts in Mathematics, vol. 152, SpringerVerlag, New York, 1995.

Mathematics Institute, University of Warwick, Coventry, CV4 7AL, United KingDOM

E-mail address: D.Maclagan@warwick.ac.uk 